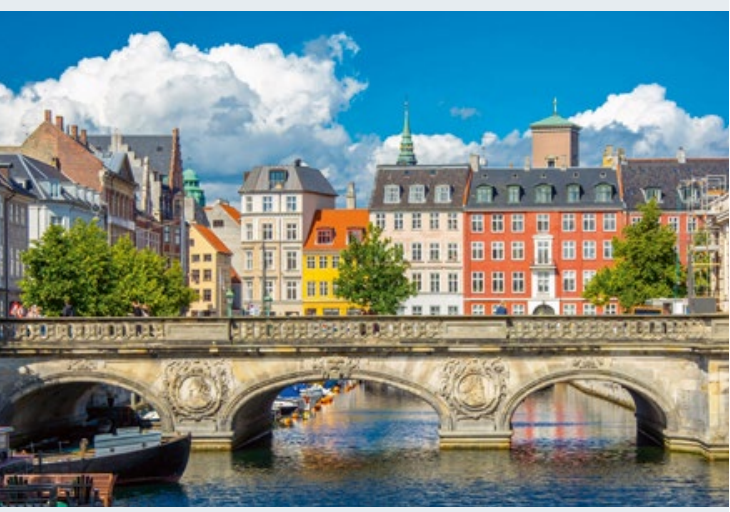

\section{EAN-Kongress zum Zweiten}

Nach ihrem Gründungskongress im letzten Jahr tagte die European Academy of Neurology, die 45 nationale europäische neurologische Gesellschaften unter ihrem Dach vereint, 2016 nun "ganz regulär" in Kopenhagen.
Medizin aktuell

8

Europäischer Blick auf die aktuelle Neurologie

Berichterstattung vom zweiten Kongress der European Academy

of Neurology vom 28. bis 31. Mai 2016 in Kopenhagen

13 Lang erwartete Antworten: S3-Leitlinie zu Diagnostik der Autismus-Spektrum-Störungen

Aus der Serie "Leitlinie im Fokus"

Literatur kompakt

16 Neue Studien zur Multiplen Sklerose

Vereinfachte MRT-Kriterien für MS - der neue

MAGNIMS-Leitfaden

Hohe JCV-Serokonversion und steigende Antikörperindizes unter Natalizumab

Malignome unter Mitoxantron: ein kalkulierbares Risiko

Kutane Nebenwirkungen unter Daclizumab häufig, aber gut behandelbar

Berechtigte Hoffnung auf die B-Zell-spezifische MS-Therapie

\section{Fortbildung Neurologie}

\section{Koffein als analgetisches Adjuvans}

Gehirndoping

Daniel Eschle, Kantonsspital Uri (Schweiz)

43 CME: Infektionen neurologischer Patienten im Alter

Atypischer Verlauf erschwert die Diagnose

Roland Nau, Marija Djukic, Göttingen, Annette Spreer, Mainz,

Helmut Eiffert, Göttingen
16 Multiple Sklerose - neue Studien

Axel Haarmann und Mathias Buttmann berichten in ihrem hochaktuellen Streifzug durch die MS-Literatur unter anderem über die Risikostratifizierung unter Natalizumab und die unangenehmen aber behandelbaren Hautveränderungen als Nebenwirkung einer Daclizumab-Gabe.

\title{
Unsere Kooperationspartner
}

\section{Editorial \\ 12 Leserbrief \\ 63 Impressum}

Titelbild: @ DAMIEN LOVEGROVE / SPL / Age

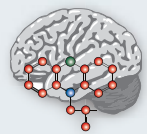

AGNP

Arbeitsgemeinschaft für Neuropsychopharmakologie und Pharmakopsychiatrie

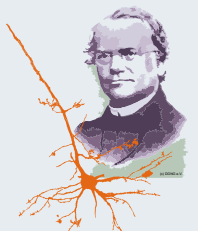

Deutsche Gesellschaft Deutsche Gesellschaft
für Neurogenetik (DGNG)

\section{bkjpp}

Berufsverband für Kinder- und Jugendpsychiatrie, Psychosomatik und Psychotherapie in Deutschland e.V. 


\section{Fortbildung Psychiatrie}

\section{Off-label bei Manie und Depression?}

Aus der Serie „Off-Label-Therapie"

Teil 4: Affektive Störungen

Heinz Grunze, Salzburg

36 CME: Voraussetzungen für eine erfolgreiche Pharmakotherapie von Depressionen

Interaktive psychoedukative Vorbereitung

Andreas Broocks, Jörg Pink, Schwerin

Praxis konkret

\section{BSG blockiert die Umgehung der öffentlichen Ausschreibung der Zulassung}

$58 \quad$ Lohnen die EBM-Kapitel 37 und 38?

Abrechnungstipps zur Vergütung der Pflegeheimbetreuung

59 Mutterschutz: Mehr Freiheit für schwangere Ärztinnen

60 Ärzte und Apotheker einigen sich auf Medikationsplan

61 eIDAS-Verordnung: Neue Regeln für die E-Signatur

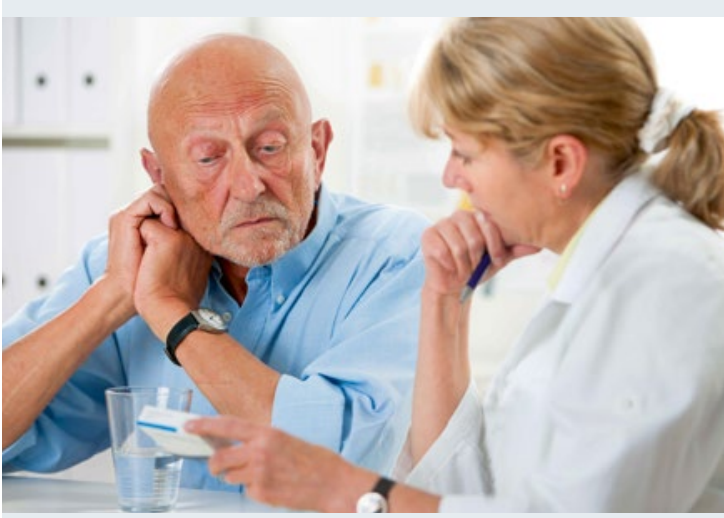

\section{Therapie der Depression}

Die Pharmakotherapie der Depression erfordert viel mehr als die reine Verordnung des richtigen Medikaments. Eine erfolgreiche Behandlung setzt vor allem auch die Klärung von Fragen und Bedenken des Patienten voraus.

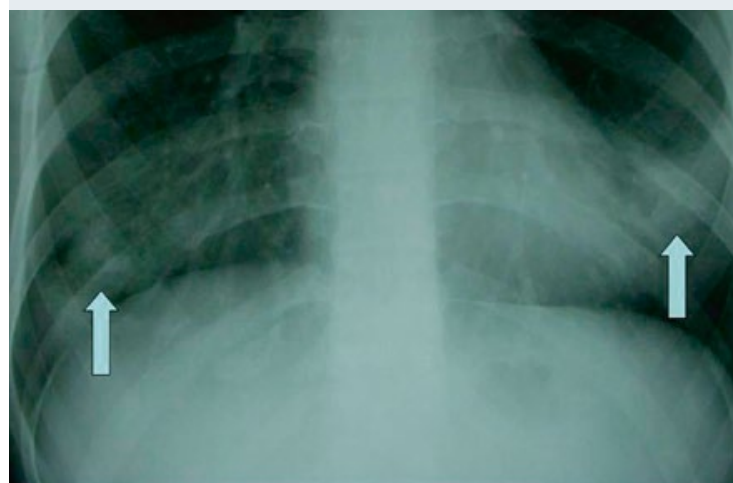

\section{Infektionen im Alter}

Infektionen im Alter sind häufig, betreffen aber seltener das Nervensystem. Dem Neurologen und Psychiater begegnen am häufigsten Harnwegs- und respiratorische Infekte. Bakterielle Meningitiden und die HS-Virus-Enzephalitis sind selten, erfordern aber eine rasche adäquate Therapie.

Parkinson-Therapie mit Piribedil

Weniger Tagesmüdigkeit, Apathie und Depression

\section{Unsere Autoren der CME-Fortbildung}

Erläutern die Voraussetzungen für eine erfolgreiche Pharmakotherapie der Depression. > Seite 36:

Prof. Dr. med. Andreas Broocks, Chefarzt der Klinik für Psychiatrie und Psychotherapie, Ärztlicher Direktor der Carl-Friedrich-Flemming-Klinik, und Dr. med. Jörg Pink, Karl-Friedrich-Flemming-Klinik Schwerin, HELIOS Kliniken Schwerin

Legen die häufigsten Infektionen neurologischer Patienten im Alter dar und erläutern die Vorgehensweise bei Diagnose, Therapie und Prävention. > Seite 43:

Prof. Dr. med. Roland Nau und PD Dr. med. Marija Djukic, Evangelisches KH GöttingenWeende und Institut für Neuropathologie, Universität Göttingen, PD Dr. med. Annette Spreer, Klinik für Neurologie, Universitätsmedizin Mainz, und Prof. Dr. med. Dr. rer. nat. Helmut Eiffert, Institut für Medizinische Mikrobiologie, Universitätsmedizin Göttingen

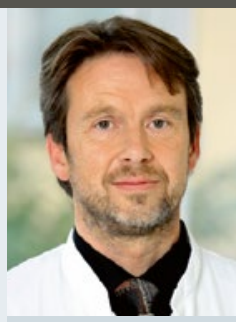

Prof. Dr. med. Andreas Broocks

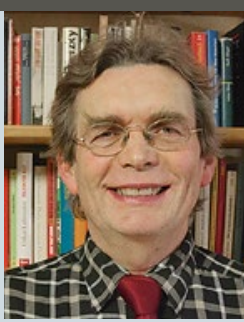

Prof. Dr. med. Roland Nau 